\title{
Comparison of the Sensititre YeastOne with CLSI M38-A2 microdilution methods for MIC determination of amphotericin B and azoles against Aspergilli
}

\author{
Hsuan-Chen Wang a, Ming-I Hsieh a, Pui-Ching Choi a , Chi-Jung Wu a,b,* (wucj@nhri.org.tw) \\ a National Institute of Infectious Diseases and Vaccinology, National Health Research Institutes, Tainan, Taiwan \\ ${ }^{b}$ Department of Internal Medicine, National Cheng Kung University Hospital, Tainan, Taiwan
}

\section{Introduction}

The 2017 ESCMID-ECMM-ERS guideline recommended performing antifungal susceptibility testing on clinically relevant Aspergillus isolates. Recently, the Sensititre YeastOne method has been increasingly adopted in both clinical and research settings for susceptibility testing of Aspergillus species. However, available studies comparing the MICs resulting from the YeastOne and CLSI methods were mostly conducted before 2008 , and azole-resistant $A$. fumigatus isolates with environmental resistance mechanisms had not been evaluated yet. Hence, this study compared the YeastOne and reference CLSI M38-A2 broth microdilution methods for antifungal susceptibility testing of Aspergillus species to investigate whether the Sensititre YeastOne method is suitable for routine use in clinical laboratories.

\section{Materials and Methods}

The minimum inhibitory concentrations (MICs) of antifungal agents against 100 isolates, comprising 31 Aspergillus fumigatus (5 $\mathrm{TR}_{34 /} \mathrm{L}$ $98 \mathrm{H}$ isolates), 23 Aspergillus flavus, 13 Aspergillus terreus, and 10 Aspergillus niger clinical isolates, and $23 \mathrm{~A}$. fumigatus environmental isolates (19 $\mathrm{TR}_{34 /} \mathrm{L} 98 \mathrm{H}$ isolates), were determined using both Sensititre YeastOne (Trek Diagnostic Systems, Ltd.) and the reference CLSI M38-A2 broth microdilution methods.

\section{Results and Discussion}

The MIC results are shown in Table 1 . The overall agreement (within two twofold dilutions) between the two methods was best for voriconazole (100\%), followed by posaconazole $(95 \%)$, itraconazole (92\%), and amphotericin B (90\%). For all isolates, the voriconazole GM MICs determined by the two methods were nearly identical ( $0.732 \mathrm{vs} .0 .758 \mathrm{mg} / \mathrm{L})$, whereas the itraconazole and posaconazole GM MICs from YeastOne were about one twofold dilution lower than those from the M38-A2 method (itraconazole 0.346 vs. $0.688 \mathrm{mg} / \mathrm{L}$; posaconazole 0.095 vs. $0.214 \mathrm{mg} / \mathrm{L})$. In contrast, the amphotericin B GM MIC was 3.3-fold higher in YeastOne than in M38-A2 (1.959 vs 0.602 $\mathrm{mg} / \mathrm{L})$.

Table 1. The MIC results for Aspergillus spp. by both methods.

\begin{tabular}{|c|c|c|c|c|c|c|}
\hline \multirow[t]{3}{*}{ Strains, no. } & \multicolumn{4}{|c|}{$\mathrm{MIC}$ (mg/L) } & \multicolumn{2}{|c|}{$\begin{array}{l}\text { \% Agreement with the } \\
\text { following twofold dilution }\end{array}$} \\
\hline & \multicolumn{2}{|c|}{ Sensititre YeastOne } & \multicolumn{2}{|c|}{ CLSI M38-A2 } & \multirow{2}{*}{ within \pm 1} & \multirow{2}{*}{ within \pm 2} \\
\hline & GM & $\mathrm{MIC}_{50} / \mathrm{MIC}_{90}$ & GM & $\mathrm{MIC}_{50} / \mathrm{MIC}_{90}$ & & \\
\hline \multicolumn{7}{|l|}{ Amphotericin B } \\
\hline All, 100 & 1.959 & $2 / 4$ & 0.602 & $0.5 / 1$ & $41(41)$ & $90(90)$ \\
\hline A. fumigatus, all, 54 & 1.828 & $2 / 2$ & 0.526 & $0.5 / 1$ & $14(25.9)$ & $50(92.6)$ \\
\hline A. flavus, 23 & 2.470 & $2 / 4$ & 1.095 & $1 / 2$ & $18(78.3)$ & $23(100)$ \\
\hline A. terreus, 13 & 1.896 & $2 / 2$ & 0.766 & $1 / 1$ & $9(69.2)$ & $13(100)$ \\
\hline A. niger, 10 & 1.741 & $2 / 2$ & 0.230 & $0.25 / 0.5$ & $0(0)$ & $4(40)$ \\
\hline All, 100 & 0.346 & $0.12 />16$ & 0.688 & $0.25 />8$ & $77(77)$ & $92(92)$ \\
\hline A. fumigatus, all, 54 & 0.906 & $0.12 />16$ & 1.644 & $0.5 />8$ & $41(75.9)$ & $50(92.6)$ \\
\hline A. flavus, 23 & 0.120 & $0.12 / 0.12$ & 0.175 & $0.12 / 0.5$ & $20(87.0)$ & $23(100)$ \\
\hline A. terreus, 13 & 0.067 & $0.06 / 0.12$ & 0.114 & $0.12 / 0.12$ & $12(92.3)$ & $13(100)$ \\
\hline A. niger, 10 & 0.186 & $0.25 / 0.25$ & 1.510 & $0.5 />8$ & $4(40)$ & $6(60)$ \\
\hline \multicolumn{7}{|l|}{ Posaconazole } \\
\hline All, 100 & 0.095 & $0.06 / 0.5$ & 0.214 & $0.12 / 1$ & $66(66)$ & $95(95)$ \\
\hline A. fumigatus, all, 54 & 0.110 & $0.06 / 0.5$ & 0.321 & $0.25 / 2$ & $27(50)$ & $50(92.6)$ \\
\hline A. flavus, 23 & 0.110 & $0.12 / 0.12$ & 0.146 & $0.12 / 0.25$ & $23(100)$ & $23(100)$ \\
\hline A. terreus, 13 & 0.048 & $0.06 / 0.06$ & 0.083 & $0.06 / 0.12$ & $10(76.9)$ & $13(100)$ \\
\hline A. niger, 10 & 0.074 & $0.06 / 0.12$ & 0.199 & $0.12 / 0.5$ & $6(60)$ & $9(90)$ \\
\hline \multicolumn{7}{|l|}{ Voriconazole } \\
\hline All, 100 & 0.732 & $0.5 / 4$ & 0.758 & $0.5 / 4$ & $100(100)$ & $100(100)$ \\
\hline A. fumigatus, all, 54 & 0.913 & $0.5 / 4$ & 0.999 & $0.5 / 4$ & $54(100)$ & $54(100)$ \\
\hline A. flavus, 23 & 0.599 & $0.5 / 1$ & 0.547 & $0.5 / 1$ & $23(100)$ & $23(100)$ \\
\hline A. terreus, 13 & 0.474 & $0.5 / 0.5$ & 0.474 & $0.5 / 0.5$ & $13(100)$ & $13(100)$ \\
\hline A. niger, 10 & 0.616 & $0.5 / 1$ & 0.660 & $0.5 / 1$ & $10(100)$ & $10(100)$ \\
\hline
\end{tabular}

Of $24 \mathrm{TR}_{34 / \mathrm{L}} \mathrm{L} 9 \mathrm{H}$ A. fumigatus isolates, itraconazole and voriconazole MICs by YeastOne correlated well with those by M38-A2 in terms of the GM MICs, the MIC ranges, the high-level agreement (within one dilution) (100\% for itraconazole and voriconazole), and categorial agreement (100\% for itraconazole and $91.7 \%$ for voriconazole). The very major error rate $(16.7 \%)$ noted for posaconazole might be explained by the observation that posaconazole YeastOne MICs were generally one dilution lower than the corresponding M38-A2 MICs.

The M38-A2 method found that four A. niger strains exhibited trailing growth with microscopically aberrant small, rounded, compact hyphal forms in wells with itraconazole concentrations ranging from 0.5 to $8 \mathrm{mg} / \mathrm{L}$, and the itraconazole MICs were read as $>8 \mathrm{mg} / \mathrm{L}$ (Figure 1 ), whereas the corresponding itraconazole MICs determined by YeastOne were all $\leq 0.5 \mathrm{mg} / \mathrm{L}$ without trailing growth. The clinical significance of trailing growth causing in vitro itraconazole resistance is not clear due to the rarity of related reports, which warrants further investigations.

\section{Conclusions}

YeastOne might be a suitable alternative method for the susceptibility testing of itraconazole, posaconazole, and voriconazoles against common Aspergillus spp. and for detecting azole-resistant $\mathrm{TR}_{34} / \mathrm{L} 98 \mathrm{H}$ A. fumigatus. Nevertheless, YeastOne tended to yield higher amphotericin B MICs and failed to detect $A$. niger isolates which exhibited trailing growth with itraconazole in the M38-A2 method. Hence, MIC interpretations in both cases should be undertaken with caution.

Figure 1. Determination of MICs of itraconazole against four Aspergillus niger strains by CLSI M38-A2. In wells with itraconazole concentrations ranging from 0.5 to $8 \mathrm{mg} / \mathrm{L},(\mathrm{A})$ trailing growth, and (B) microscopically aberrant growth (small, rounded, compact hyphal forms, C01-014) are illustrated. (A)

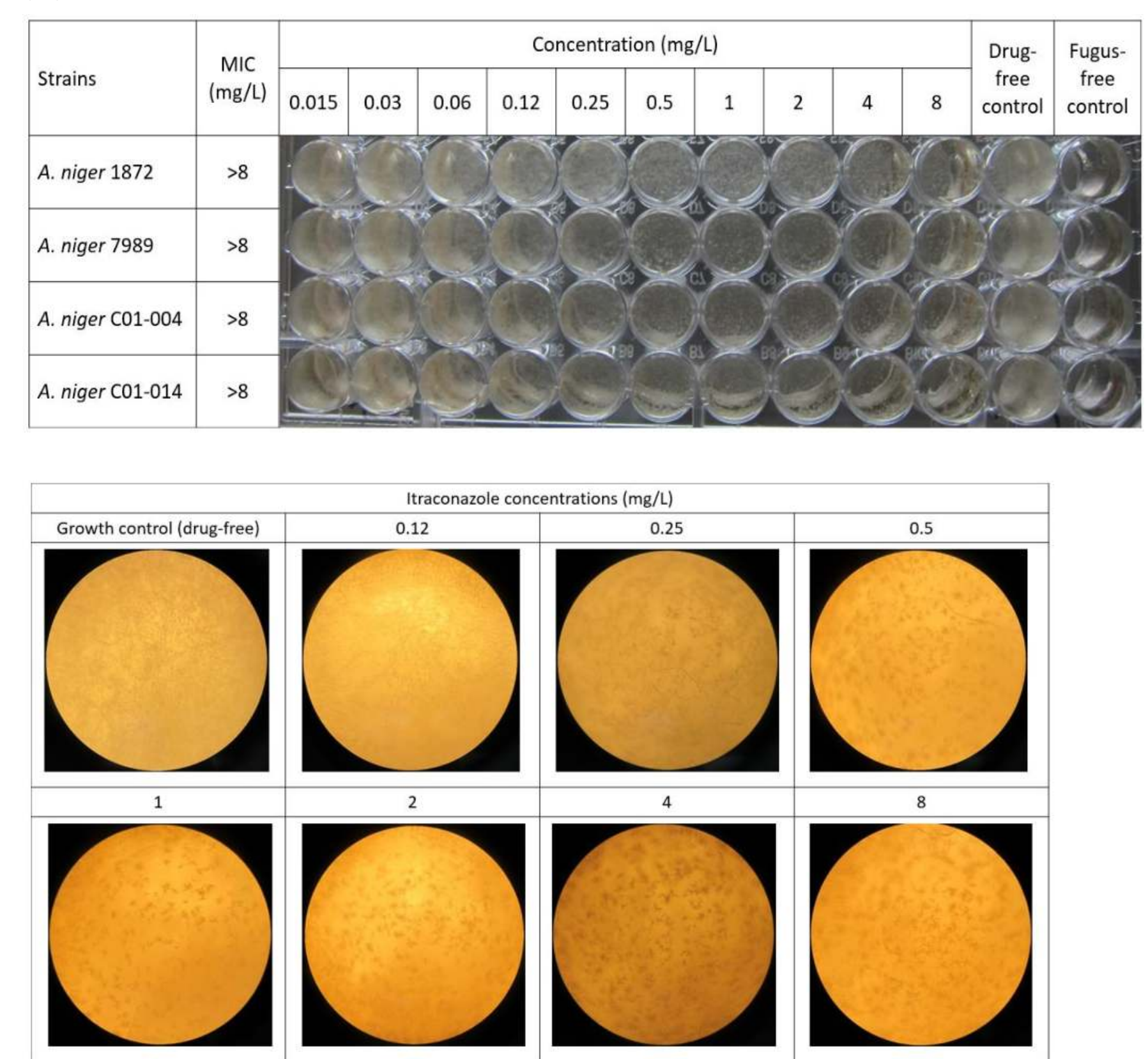

